\title{
Neu anerkannte bzw. neu eingeteilte Weiterbildungsstätten Etablissements nouvellement reconnus resp. classés pour la formation postgraduée
}

Mit der nachstehenden Publikation setzt der Zentralvorstand der Verbindung der Schweizer Ärztinnen und Ärzte FMH die Neuanerkennungen, Ein- bzw. Umteilungen der nachfolgend aufgeführten Weiterbildungsstätten/Arztpraxen per 1. Juli 2000 in Kraft.

Par la présente publication, le Comité central de la Fédération des médecins suisses FMH procède, au $1^{\text {er }}$ juillet 2000, à la reconnaissance et à la classification ou au changement de catégorie des établissements de formation et des cabinets médicaux ciaprès.

\section{Weiterbildungsstätten}

\section{Allergologie und klinische Immunologie}

Bern, Klinik für Rheumatologie und Klinische Immunologie/Allergologie, Inselspital Kategorie Aa/Ai (2 Jahre), Umteilung von Kategorie Bi (1 Jahr) in Kategorie Aa/Ai (2 Jahre) im Rahmen eines Chefarztwechsels

Faltigberg, Zürcher Höhenklinik Wald Kategorie Ca (6 Monate), Neuanerkennung

Zürich, Abteilung Immunologie/Hämatologie, Kinderspital Zürich

Kategorie $\mathrm{Aa}^{*} / \mathrm{Ai}$ (2 Jahre), Bestätigung der Anerkennung

\section{Allgemeinmedizin}

Basel, Klinik Hildegard Hospiz, Spezialklinik für palliative Medizin und Nachbehandlung von Akutpatienten

Kategorie Disziplinen zur freien Wahl (anerkannt für 1 Jahr), Neuanerkennung

Sorengo, Clinica S. Anna

Kategorie Disziplinen zur freien Wahl (anerkannt für 1 Jahr), Bestätigung der Anerkennung im Rahmen eines Chefarztwechsels

\footnotetext{
Anästhesiologie

Wetzikon, Abteilung Anästhesie und Reanimation, Schwerpunktspital

Kategorie C (1 Jahr), Neuanerkennung
}

\section{Arbeitsmedizin}

Zürich, Institut für Hygiene und Arbeitsphysiologie ETH

Kategorie A (1 Jahr), Bestätigung der Anerkennung im Rahmen eines Chefarztwechsels

Zürich, Überbetrieblicher arbeitsmedizinischer Dienst, AEH Zentrum für Arbeitsmedizin, Ergonomie und Hygiene $\mathrm{GmbH}$

Kategorie B (1 Jahr bzw. 2 Jahre), Neuanerkennung

\section{Chirurgie}

Chur, Chirurgische Abteilung, Kreuzspital Kategorie BI (3 Jahre), Bestätigung der Anerkennung im Rahmen eines Chefarztwechsels

Dornach, Chirurgische Klinik, Spital Dornach Kategorie BII (2 Jahre), Bestätigung der Anerkennung im Rahmen eines Chefarztwechsels

Rüti, Klinik für Chirurgie, Spital Rüti-Wald Kategorie BI (3 Jahre), Bestätigung der Anerkennung im Rahmen eines Chefarztwechsels

Sierre, Service de chirurgie, Hôpital de Sierre-Loèche Kategorie BI (3 Jahre), Bestätigung der Anerkennung im Rahmen eines Chefarztwechsels

St-Imier, Service de chirurgie, Hôpital du district de Courtelary

Kategorie BII (2 Jahre), Bestätigung der Anerkennung im Rahmen eines Chefarztwechsels

\section{Gastroenterologie}

Aarau, Abteilung Gastroenterologie, Kantonsspital Kategorie B (2 Jahre), Bestätigung der Anerkennung im Rahmen der Reevaluation der FAGAS

Basel, Gastroenterologie Nordwestschweiz, Kantonsspital Basel

Kategorie A (3 Jahre), Bestätigung der Anerkennung im Rahmen der Reevaluation der FAGAS 
Bern, Gastroenterologische Abteilung, Inselspital Kategorie A (3 Jahre), Bestätigung der Anerkennung im Rahmen der Reevaluation der FAGAS

Genève, Division de gastroentérologie, Dép. médecine interne, HUG

Kategorie A (3 Jahre), Bestätigung der Anerkennung im Rahmen der Reevaluation der FAGAS

Lausanne, Service de gastroentérologie, Dép. médecine interne, CHUV

Kategorie A (3 Jahre), Bestätigung der Anerkennung im Rahmen der Reevaluation der FAGAS

Luzern, Abteilung Gastroenterologie, Kantonsspital Kategorie B (2 Jahre), Bestätigung der Anerkennung im Rahmen der Reevaluation der FAGAS

St. Gallen, Gastroenterologische Abteilung Klinik C, Kantonsspital

Kategorie B (2 Jahre), Bestätigung der Anerkennung im Rahmen der Reevaluation der FAGAS

Winterthur, Abteilung Gastroenterologie, Kantonsspital

Kategorie B (2 Jahre), Bestätigung der Anerkennung im Rahmen der Reevaluation der FAGAS,

Provisorische Anerkennung bis 31.12.2002!

Zürich, Abteilung für Gastroenterologie, Departement Innere Medizin, USZ

Kategorie A (3 Jahre), Bestätigung der Anerkennung im Rahmen der Reevaluation der FAGAS

Zürich, Gastroenterologische Abteilung, Medizinische Klinik, Stadtspital Triemli Kategorie B (2 Jahre), Bestätigung der Anerkennung im Rahmen der Reevaluation der FAGAS

Zürich, Abteilung Gastroenterologie, Medizinische Klinik, Stadtspital Waid Kategorie B (2 Jahre), Bestätigung der Anerkennung im Rahmen der Reevaluation der FAGAS

\section{Gynäkologie und Geburtshilfe}

Couvet, Service de gynécologie et obstétrique, Hôpital du Val-de-Travers Kategorie D (1 Jahr), Neuanerkennung

Walenstadt, Gynäkologisch-geburtshilfliche Klinik, Kantonales Spital Walenstadt

Kategorie D (1 Jahr), Bestätigung der Anerkennung im Rahmen eines Chefarztwechsels

\section{Hals- und Gesichtschirurgie}

Zürich, ORL, Hals- und Gesichtschirurgie, USZ anerkannt für 2 Jahre, Bestätigung der Anerkennung im Rahmen eines Chefarztwechsels

\section{Hämatologie}

Aarau, Blutspendezentrum SRK, Zentrum für Onkologie, Hämatologie und Transfusionsmedizin, Kantonsspital Aarau

Kategorie D1 (1 Jahr)/Blutspendezentren,

Bestätigung der Anerkennung im Rahmen eines Chefarztwechsels

Luzern, Abteilung Hämatologie, Kantonsspital Kategorie B (2 Jahre)/Klinische Hämatologie, Bestätigung der Anerkennung im Rahmen eines Chefarztwechsels

Zürich, Transfusionslabor, Abt. Hämatologie/ Dept. Innere Medizin, USZ

Kategorie D1 (1 Jahr)/Blutspendezentren,

Bestätigung der Anerkennung im Rahmen eines Chefarztwechsels

\section{Herz- und thorakale Gefässchirurgie}

Bern, Herzzentrum, Klinik Beau-Site

Kategorie B (1 Jahr), Bestätigung der Anerkennung im Rahmen eines Chefarztwechsels

Bern, Klinik für Thorax-, Herz- und Gefässchirurgie, Inselspital

Kategorie A (4 Jahre), Bestätigung der Anerkennung im Rahmen eines Chefarztwechsels

\section{Infektiologie}

Basel, HIV-Sprechstunde der Medizinischen

Universitäts-Poliklinik, Kantonsspital

Kategorie B (1 Jahr), Neuanerkennung

\section{Innere Medizin}

Carouge, Permanence médico-chirurgicale, Clinique de Carouge

Kategorie C (1 Jahr), Neuanerkennung

Gais, Fachklinik für kardiale und psychosomatische Rehabilitation, Klinik Gais AG

Kategorie C (1 Jahr), Aufhebung der provisorischen Anerkennung und Bestätigung in Kategorie C

Laufen, Medizinische Abteilung,

Kantonsspital Laufen

Kategorie C (1 Jahr), Umteilung von Kategorie D in Kategorie C im Rahmen eines Chefarztwechsels

Liestal, Medizinische Universitätsklinik, Kantonsspital

Kategorie A (3 Jahre), Bestätigung der Anerkennung im Rahmen eines Chefarztwechsels

Lugano, Clinica al Parco SA

Kategorie D (6 Monate), Bestätigung der Anerkennung im Rahmen eines Chefarztwechsels 
Neuchâtel, Service de médecine,

Hôpital de la Ville aux Cadolles

Kategorie A (3 Jahre), Bestätigung der Anerkennung im Rahmen eines Chefarztwechsels

Orselina, Clinica Fondazione G. Varini

Kategorie D (6 Monate), Bestätigung der Anerkennung im Rahmen eines Chefarztwechsels

Samedan, Medizin, Spital Oberengadin

Kategorie B (2 Jahre), Bestätigung der Anerkennung im Rahmen eines Chefarztwechsels

St-Maurice, Clinique St-Amé

Kategorie C (1 Jahr), Umteilung von Kategorie D

(6 Monate) in Kategorie C (1 Jahr)

Tafers, Medizinische Abteilung,

Spital des Sensebezirks

Kategorie C (1 Jahr), Bestätigung der Anerkennung im Rahmen eines Chefarztwechsels

\section{Kardiologie}

Bellinzona, Servizio di cardiologia,

Ospedale San Giovanni

Kategorie B (2 Jahre), Neuanerkennung

\section{Kardiologie zu Kinder- und Jugendmedizin}

Bern, Abteilung für Kardiologie,

Med. Kinderklinik und Poliklinik, Inselspital

Kategorie B (1 Jahr), Bestätigung der Anerkennung im Rahmen eines Chefarztwechsels

\section{Kinderchirurgie}

Luzern, Kinderchirurgie, Kantonsspital Luzern Kategorie A ( $3^{1 / 2}$ Jahre), Bestätigung der Anerkennung im Rahmen eines Chefarztwechsels

\section{Kinder- und Jugendmedizin}

Biel, Kinderspital Wildermeth

Kategorie B (2 Jahre), Bestätigung der Anerkennung im Rahmen eines Chefarztwechsels

\section{Kinder- und Jugendpsychiatrie und -psychotherapie}

Bern, Kinder- und jugendpsychiatrischer Dienst BielSeeland/Berner Jura mit Liaisonpsychiatrischem Dienst an der Kinderklinik des Spitalzentrums Biel Kategorie A (4 Jahre), Fusion mit der Kinder- und Jugendpsychiatrischen Klinik in Bern

\section{Klinische Zytopathologie}

Aarau, Institut für histologische und zytologische Diagnostik Kategorie (6 Monate) / Weiterbildungsstätten für Klinische Zytopathologie, Neuanerkennung

Bern, Labor Dr. Denzler, Gynäkologische Zytologie Kategorie D (3 - 6 Monate)/Weiterbildungsstätten für Gynäkologische Zytologie, Neuanerkennung

Münsterlingen/Scherzingen, Zytopathologie, Institut für Pathologie, Thurgauisches Kantonsspital Kategorie B (12 Monate)/Gemischte Weiterbildungsstätten, Bestätigung der Anerkennung im Rahmen eines Chefarztwechsels

\section{Medizinische Radiologie / Radiodiagnostik}

Sion, Radiodiagnostic et médecine nucléaire, Hôpital régional Sion-Hérens-Conthey Kategorie A (4 Jahre), Umteilung von Kategorie B (3 Jahre) in Kategorie A (4 Jahre)

\section{Medizinische Radiologie / Nuklearmedizin}

Bellinzona, Reparto medicina nucleare e radioterapia oncologica, Ospedale San Giovanni

Kategorie B (2 Jahre), Bestätigung der Anerkennung im Rahmen eines Chefarztwechsels

Zürich, Klinik für Nuklearmedizin und Radiotherapie, Stadtspital Triemli

Kategorie B (2 Jahre), Bestätigung der Anerkennung im Rahmen eines Chefarztwechsels

\section{Medizinische Radiologie / Radio-Onkologie}

Zürich, Klinik für Nuklearmedizin und Radiotherapie, Stadtspital Triemli

Kategorie A (4 Jahre), Umteilung von Kategorie B (2 Jahre) in Kategorie A (4 Jahre)

\section{Nephrologie}

Biel, Nephrologie/Hämodialyse, Regionalspital Biel Kategorie B (1 Jahr), Umteilung von Kategorie C (6 Monate) in Kategorie B (1 Jahr)

Lugano, Reparto nefrologia e emodialisi, Ospedale Regionale Lugano

Kategorie B (1 Jahr), Umteilung von Kategorie C (6 Monate) in Kategorie B (1 Jahr)

St. Gallen, Nephrologie, Medizinische Klinik B, Kantonsspital

Kategorie A (3 Jahre), Umteilung von Kategorie B (1 Jahr) in Kategorie A (3 Jahre) im Rahmen eines Chefarztwechsels 


\section{Neurochirurgie}

Winterthur, Neurochirurgische Abteilung, Kantonsspital Winterthur

Kategorie C (1 Jahr), Bestätigung der Anerkennung im Rahmen eines Chefarztwechsels

\section{Ophthalmologie}

Olten, Klinik Pallas AG

Kategorie C1 (2 Jahre), Neuanerkennung, Provisorische Anerkennung bis 30.6.2004!

\section{Ophthalmologie, spez. Ophthalmochirurgie}

Olten, Klinik Pallas AG

Kategorie D2 (1 Jahr), Neuanerkennung, Provisorische Anerkennung bis 30.6.2004!

\section{Orthopädische Chirurgie}

Novaggio, Clinica federale di riabilitazione Kategorie C (1 Jahr), Neuanerkennung

\section{Oto-Rhino-Laryngologie}

Zürich, ORL, Hals- und Gesichtschirurgie, USZ Kategorie A (3 Jahre), Bestätigung der Anerkennung im Rahmen eines Chefarztwechsels

\section{Pathologie}

Bern, Pathologie Länggasse

Kategorie C (2 Jahre), Neuanerkennung

\section{Pädiatrische Radiologie}

Winterthur, Radiologisches Institut, Kantonsspital Kategorie B (1 Jahr), Neuanerkennung

\section{Pharmazeutische Medizin}

Glattbrugg, Medical Departement, Merck Sharp \& Dohme-Chibret AG Kategorie A (3 Jahre), Neuanerkennung

Vernier, Medizinische Abteilung, Eli Lilly (Suisse) SA Kategorie B (2 Jahre), Neuanerkennung

Zürich, Pharmapart AG

Kategorie B (2 Jahre), Neuanerkennung

\section{Phoniatrie}

Luzern, Phoniatrische Abteilung, ORL-Klinik, Kantonsspital

voll anerkannt, Bestätigung der Anerkennung im Rahmen eines Chefarztwechsels

\section{Physikalische Medizin und Rehabilitation}

Bern, Klinik für Rheumatologie und Klinische Immunologie/Allergologie

Kategorie A1 (2 Jahre), Bestätigung der Anerkennung im Rahmen eines Chefarztwechsels

Davos Clavadel, Physikalische Medizin \& Rehabilitation, Zürcher Höhenklinik Davos Kategorie A2 (1 Jahr), Neuanerkennung

\section{Plastische und Wiederherstellungschirurgie}

Basel, Abteilung für Plastische und Rekonstruktive Chirurgie, Klinik für Wiederherstellende Chirurgie, Kantonsspital

Kategorie A (4 Jahre), Bestätigung der Anerkennung im Rahmen eines Chefarztwechsels

\section{Pneumologie zu Kinder- und Jugendmedizin}

Basel, Pneumologie, Universitäts-Kinderspital beider Basel

Kategorie A (3 Jahre), Bestätigung der Anerkennung im Rahmen eines Chefarztwechsels

Genève, Pneumologie pédiatrique, Département de Pédiatrie, HUG

Kategorie A (3 Jahre), Umteilung von Kategorie B (2 Jahre) in Kategorie A (3 Jahre)

\section{Psychiatrie und Psychotherapie}

Chur, Ambulanter sozialpsychiatrischer Dienst, Kantonale Psychiatrische Klinik Waldhaus Kategorie CI (2 Jahre), Einteilung gemäss Weiterbildungsprogramm vom 1.1.1998

Littenheid, Klinik für Psychiatrie und Psychotherapie Kategorie BI (3 Jahre)/stationär, Umteilung von Kat. BI (3 Jahre)/gemischt in Kategorie BI (3 Jahre)/ stationär

San Pietro, Clinica San Pietro

Kategorie CI (2 Jahre) / stationär, Neuanerkennung

Sirnach, Externer Psychiatrischer Dienst,

Klinik für Psychiatrie \&t Psychotherapie

Kategorie CI (2 Jahre) / ambulant, Neuanerkennung

Winterthur, SOMOSA, Sozialpädagogischpsychiatrische Modellstation

Kategorie DI (2 Jahre) / stationär, Einteilung gemäss Weiterbildungsprogramm vom 1.1.1998 


\section{Urologie}

Biel, Abteilung für Urologie, Regionalspital Kategorie B (1 Jahr), Bestätigung der Anerkennung im Rahmen eines Chefarztwechsels/ Provisorische Anerkennung bis 31.12.2000!

Genève, Clinique d'Urologie, HUG Kategorie A (4 Jahre), Bestätigung der Anerkennung im Rahmen eines Chefarztwechsels

\section{Liste der Weiterbildungsstätten für Innere Medizin mit geriatrischer, rehabilitativer oder polyvalenter Ausrichtung*}

\section{Liste des établissement de formation postgraduée pour la médecine interne avec orientation gériatrique, réadaptive ou polyvalente}

Aubonne, Service de médecine, Hôpital régional

Epalinges, CUTR Cevey-Sylvaplana

Genève, Unité de la division de médecine pénitentiaire l'IUML, Service médical à la prison de Champ-Dollon

Hasliberg Hohfluh, REHA-Klinik Hasliberg

Heiligenschwendi, Berner REHA Zentrum Heiligenschwendi

Lausanne, Unité de médecine du personnel, Div. autonome de médecine préventive hospitalière, CHUV

Leysin, Centre de traitement et de réadaptation, Clinique Miremont CTR
Montana-Vermala, Intern-medizinische Spitalabteilung, Berner Klinik Montana

Orselina, Clinica Fondazione G. Varini

Schinznach Bad, Rheuma- und Rehabilitationsklinik Sementina, Centro Riabilitazione Sementina

St-Maurice, Clinique St-Amé

Zürich, Klinik für Akut-Geriatrie, Bircher Klinik Susenberg

* Kann im Rahmen der Weiterbildung zum Arzt für Allgemeinmedizin FMH höchstens für 6 bzw. 12 Monate (Kategorie B + C: 12 Monate, Kategorie D: 6 Monate) und nur für das zweite internmedizinische Pflichtjahr angerechnet werden. 


\section{Neue Arztpraxen / Nouveaux cabinets médicaux}

\section{Allgemeinmedizin}

Biglen, Dr. med. Hanspeter Abbühl

Bürglen, Dr. med. Olivier Kappeler

Cazis, Dr. med. Walter Huber

Courtepin, Dr Monique Monney-Faller

Langnau i. E., Dr. med. Hansueli Albonico

Luzern, Dr. med. Daniel Matter/

Rückwirkende Anerkennung per 1.8.1999!

Muri AG, Frau Dr. med. Verena Gantner

Onex, Dr Dave Baer

Saas Fee, Dr. med. Philipp Salzmann

Schattdorf, Dr. med. Paul von Buren

Sta. Maria V.M, Dr. med. Matthias Christof Furrer

Vevey, Dr Juerg Rolf Eidenbenz

Wettingen, Dr. med. Martin Knaus

Winterthur, Dr. med. Urs Aemissegger

Zofingen, Dr. med. Walter Regli/

Rückwirkende Anerkennung per 1.3.2000!

Dermatologie und Venerologie

Rorschach, Dr. med. Martin Widmer

\section{Innere Medizin}

Bremgarten b. Bern, Dr. med. Juerg Bodmer

Luzern, Dr. med. Urs Sonntag

Mendrisio, Dr. med. Gianfranco Bolognini/

Rückwirkende Anerkennung per 1.7.1999!

Oron-la-Ville, Dr Michaël Klay

Schaffhausen, Dr. med. Ueli Haag

Winterthur, Dr. med. Georg Martin Stoffel

\section{Kinder- und Jugendmedizin}

Basel, Frau Dr. med. Savita Khanna/

Anerkennung ab 1.8.2000!

Interlaken, Dr. med. Helene Agnes Hochreutener

Zürich, Dr. med. Walter Jörg

\section{Ophthalmologie}

Altdorf, Dr. med. Roman 0susky

Bülach, Dr. med. Antonius Hegyaljai

Olten, Dr. med. David Pestalozzi

Wil, Dr. med. Vratislav Partyngl

\section{Ophthalmologie, spez. Ophthalmochirurgie}

Bülach, Dr. med. Antonius Hegyaljai

Wil, Dr. med. Vratislav Partyngl

Plastische und Wiederherstellungschirurgie

Lausanne, PD Dr Gaston-François Maillard

\section{Rheumatologie}

Dussnang, Dr. med. Hansruedi Gugg 


\section{Folgende Weiterbildungsstätten wurden von der Liste gestrichen}

\section{Les établissements de formation postgraduée suivants ont été supprimés de la liste}

\section{Chirurgie}

Châtel-St-Denis, Service de chirurgie, Hôpital Monney de District (Abteilung wurde geschlossen!)

Grosshöchstetten, Chirurgie, Bezirksspital (Spital wurde geschlossen!)

Herzogenbuchsee, Chirurgie, Bezirksspital (Spital wurde geschlossen!)

Jegenstorf, Chirurgie, Bezirksspital Fraubrunnen (Spital wurde geschlossen!)

Oberdiessbach, Chirurgie, Regionales Spitalzentrum Aare-/Kiesental

(Abteilung wurde geschlossen!)

Sumiswald, Abteilung Chirurgie, Bezirksspital (Abteilung wurde geschlossen!)

\section{Gynäkologie und Geburtshilfe}

Châtel-St-Denis, Service de gynécologie et obstétrique, Hôpital Monney de District (Abteilung wurde geschlossen!)

Jegenstorf, Gynäkologie und Geburtshilfe, Bezirksspital Fraubrunnen (Spital wurde geschlossen!)

Monthey, Service de gynécologie et obstétrique, Hôpital du Chablais (Aufgrund der Fusion mit dem Spital in Aigle ist in Monthey keine Assistentenstelle mehr vorhanden!)

Sumiswald, Abteilung Gynäkologie/Geburtshilfe, Bezirksspital

(Abteilung wurde geschlossen!)

\section{Hämatologie}

Locarno, Laboratorio di ricerca del Servizio oncologico cantonale, Ospedale regionale (Integration in Istituto oncologico della Svizzera Italiana, Ospedale San Giovanni, Bellinzona!)

\section{Handchirurgie}

St. Gallen, Zentrum St. Leonhard, Klinik für Hand- und ambulante Chirurgie (Möchte von der Liste der anerkannten Weiterbildungsstätten gestrichen werden!)

\section{Herz- und thorakale Gefässchirurgie}

Zürich, Klinik im Park

(Keine reguläre Weiterbildungsstelle vorhanden!)

\section{Innere Medizin}

Aigle, Service de médecine interne, Hôpital du Chablais

(Aufgrund der Fusion mit dem Spital in Monthey wird in Aigle keine Innere Medizin mehr angeboten!)

Grosshöchstetten, Medizin, Bezirksspital (Spital wurde geschlossen!)

Herzogenbuchsee, Medizinische Abteilung, Bezirksspital

(Spital wurde geschlossen!)

Jegenstorf, Medizinische Abteilung,

Bezirksspital Fraubrunnen

(Spital wurde geschlossen!)

Sumiswald, Abteilung Innere Medizin, Bezirksspital (Abteilung wurde geschlossen!)

\section{Intensivmedizin}

Bern, Medizinische Intensivstation, Inselspital (Die Medizinische Intensivstation wurde mit der Abteilung für Intensivbehandlung zusammengelegt!) 
Kinder- und Jugendpsychiatrie und -psychotherapie

Biel, Abteilung für Kinder- und Jugendpsychiatrie, Kinderklinik Wildermeth

(Fusion mit der Kinder- und Jugendpsychiatrischen Klinik in Bern!)

Ennetbaden, KTS Kinderpsychiatrische Therapiestation Ennetbaden/Koblenz

(Möchte von der Liste der anerkannten

Weiterbildungsstätten gestrichen werden!)

\section{Medizinische Genetik}

Bern, Medizinische Genetik, Medizinische Universitätskinderklinik, Inselspital (Möchte von der Liste der anerkannten Weiterbildungsstätten gestrichen werden!)

\section{Physikalische Medizin und Rehabilitation}

Sion, Service de médecine physique et réadaptation et rhumatologie, Hôpital régional Sion-HérensConthey

(Ist neu in der Clinique Romande de Réadaptation in Sion integriert!)

\section{Rheumatologie}

Sion, Service de médecine physique et réadaptation et rhumatologie, Hôpital régional Sion-HérensConthey

(Ist neu in der Clinique Romande de Réadaptation in Sion integriert!)

\title{
Folgende Arztpraxis wird von der Liste gestrichen
}

\author{
Innere Medizin
}

Effretikon, Dr. med. Sigmund Baumöhl

(Möchte von der Liste gestrichen werden!) 